\title{
Solution to Travelling Salesman Problem by Nature Inspired Algorithm
}

\author{
Sarita Rai \\ Lakshmi Narain College of Technology, \\ India
}

\author{
Rajkumar Sharma \\ Lakshi Narain College of Technology, \\ India
}

\begin{abstract}
Travelling salesman problem (TSP) finds applications in wide domains. It is a well known NP Hard problem. In this paper we have proposed nature inspired ant colony optimization on travelling salesman problem. A comparative analysis is done among the standard travelling salesman problem and proposed ant colony based approaches. All the implementations are compared in terms of communication overhead and execution time. It is found that multi colony based approach in which all colonies are independent is most efficient in terms of communication overhead and execution time.
\end{abstract}

\section{Keywords}

Travelling salesman problem, Ant colony optimization, NPHard, metaheuristic.

\section{INTRODUCTION}

NP-Hard problems has been the centre of attraction to the researchers. The algorithms which can't be solved in polynomial time by exact algorithms belongs to class of NPHard problems. In this paper the algorithms aimed at is travelling salesman problem. Many approaches to solve TSP are proposed in the past. In this paper TSP is solved using a metaheuristic algorithm called Ant colony optimization (ACO)[3,7]. There are many variants of ACO discussed in this paper and multi colony and single colony approaches exists in every variant. We have applied max min ant system on TSP.

\subsection{Travelling Salesman Problem}

In Travelling salesman problem [4][2] , a salesman has to construct a tour from his hometown to different cities such that he should visit every city exactly once and total tour length should be minimum. Such type of problem can be suitably represented using graphs. Given a Graph $\mathrm{G}$ in which vertices will represent cities and edges will represent distance between the respective cities. We have to find a tour starting from a vertex such that all the vertices of the graph should be visited exactly once before returning to initial vertex.

So travelling salesman problem is actually a problem to find Hamiltonian circuit within the graph $G$ which is a NP complete problem.

\section{Constraints in travelling salesman problem :}

- Tour length should me minimum

- And each vertex should be visited exactly once.

\section{METAHEURISTC ALGORITHM}

There doesn't exists polynomial bound algorithm for NP-Hard problems. These type of problems generate large instances and thus search space is very large for such problems. Thus approximate methods are used to return near optimal solution to such NP-Hard problems. Suh type of algorithms are called heuristic. The algorithm to find exact solution will solve it in exponential time. To NP Hard problems two types of solutions exist :

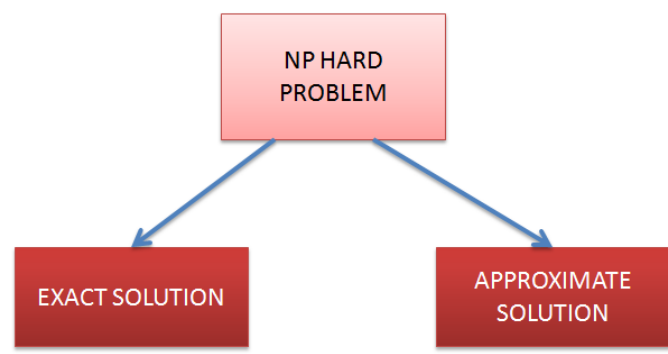

Figure 1 : approaches to solve NP-Hard problems

\section{a. Exact solution :}

There exists exact solution to NP-Hard problems but these take exponential order time. So it become impractical to use such algorithms for large problem instances such as large graph in case of travelling salesman problem.

\section{b. Approximate solution :}

Approximate algorithms return near optimal solution to such problems. The algorithms use some problem specific knowledge to improve their solution in every iteration such algorithms are called metaheuristic algorithm. There are numerous metaheuristic algorithms like migrating bird optimization, firefly optimization, Ant colony optimization and others. In this paper we have applied ACO on TSP.

\section{ANT COLONY OPTIMIZATION}

There are many variants of ACO. These are as follows :

- $\quad$ Ant system (AS).

- $\quad$ Elitist AS.

- Ant-Q

- Ant colony system.

- Max-Min AS.

- Rank based AS.

- ANTS

- Hyper-cube AS. 


\section{MAX-MIN ANT SYTEM}

Ant colony optimization[1][2][6] was proposed in early 90's. The first algorithm proposed was Ant system (AS) by Dorigo subsequent to which many variants were proposed.Among all of them Max-Min Ant system provides best results in most of the cases. All the variants use a general concept in which stochastic state transition rules which are function of pheromone value and heuristic information and two other parameters $\alpha$ and $\beta$ are used. $\alpha$ and $\beta$ are used to control the effects of pheromone and heuristic information so that in different scenarios we can adjust these two values accordingly and obtain the desired optimal result.

There are two main phases of every Ant inspired algorithm. These are :-

- Solution construction

- Pheromone update

a) Solution construction : Ant select next node to be cited using a stochastic rule. Ant ' $\mathrm{k}$ ' at node ' $\mathrm{i}$ ' has the following probability to select node $j$ as next node at time ' $t$ '

$$
p_{i, j}^{k}=\frac{\left[\tau_{i, j}\right]^{\alpha}\left[\eta_{i, j}\right]^{\beta}}{\sum_{l \in N_{i}^{k}}\left[\tau_{i, l}\right]^{\alpha}\left[\eta_{i, l}\right]^{\beta}}, \quad \text { if } j \in N_{i}^{k}
$$

Where,

$p_{i j}^{k}=$ Probability with which ant k moves from i node to j.

$\eta_{i j}=$ heuristic information.

$\tau_{i j}(t)=$ pheromone trail update.

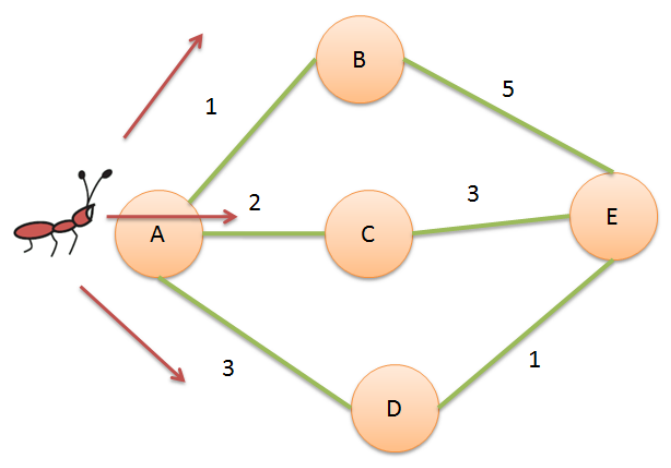

Figure 2 : illustrating ant probabilistic approach for a graph to choose next city to visit.

And this heuristic information depends on the edge length. $\alpha$ and $\beta$ are adjustable parameters to adjust relative influence of probability and heuristic information.

b) Pheromone update : After all ants have constructed their tours pheromone trails are updated.

This is done by first lowering the pheromone value on all edges by a constant factor called evaporation rate :

$$
\tau_{i j} \leftarrow(1-\rho) \cdot \tau_{i j}, \text { for all }(\mathrm{i}, \mathrm{j}) \in \mathrm{L}
$$

After evaporation all ants deposits pheromones on the edges they have visited in their tours.

$$
\tau_{i j} \leftarrow \tau_{i j}+\sum_{k=1}^{m} \Delta \tau_{i j}{ }^{k} \text {, for all }(\mathrm{i}, \mathrm{j}) \in \mathrm{L}
$$

Where,

$$
\Delta \tau_{i j}{ }^{k}=\left\{\begin{array}{cl}
1 / C_{k}, & \text { if }(i, j) \in T_{k}(t) \\
0, & \text { otherwise }
\end{array}\right.
$$

Where $C_{k}$ is the length of the tour $\mathrm{T}^{\mathrm{k}}$ built by ant $\mathrm{k}$ having the best optimal solution.

In Ant System the above process which comprises of solution construction and pheromone update is for a colony of ants. If there are more than one colonies of ants then both the colonies will have one local pheromone update matrix and will share one global pheromone update matrix.

\section{RELATED WORK}

ACO has been the attraction point of researchers over last decade. The performance of ACO depends on the termination condition being selected. In [6] they have predicted best termination condition for ACO. This prediction is done from the solved instances of the problem having best results. Then this predicted termination condition is applied over new instances of problem. In [10] ACO has been applied on cloud task scheduling which is also a NP-Hard problem. ACO is used to predict to which VM incoming cloudlet should be submitted to balance the load and efficient utilization of cloud resources. In [4] authors have applied ACO on dynamic traffic planning approach to balance the traffic among the possible travel paths available between a source and destination.

In [3] self adaptive approach for ACO to TSP is proposed so as to avoid local optima problem. In [2] multi objective problems has been solved using ACO for multi variables.

The proposed scheme in this paper focus on single colony and multi colony approach using Max-Min Ant system for Travelling salesman problem.

\section{ANT COLONY APPROACHES}

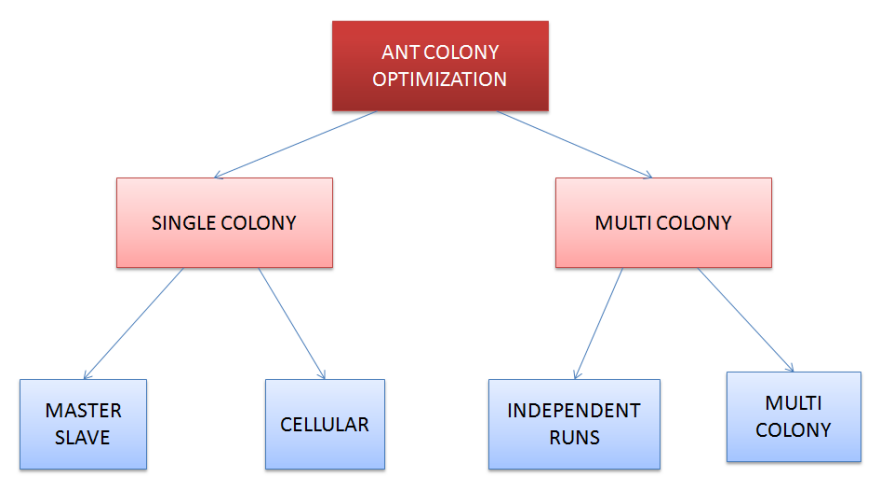

Figure 3 : colony based approaches to ACO

\section{Strategies for information exchange :}

Single colony approaches : In single colony ant system information regarding shortest tour can be exchange in two modes :

a. Local best solution : In this approach all the ants share their local best solutions among themselves in every iteration and all updates their local 
pheromone trails. Such approach may lead to stagnation and results in poor results.

b. Global best solution : In this approach global best solution within an iteration in selected and only that trail having shortest tour length is updated. This minimizes stagnation.

Multi colony approaches : In multi colony ant system multiple ant colonies search for the best solution and explore the search space. In this approach colonies can interact with each other in 2 ways :

a. Exchange of globally best solution : In this approach ant colonies shares solutions after the iteration and global best solution is shared among all. In this approach interaction is less and thus overhead of communication is minimum.

b. Circular exchange of local best solution : In this approach communication overhead is more as all the local solutions are exchanged but in some cases it gives better results.

\section{EXPERIMANTAL SETUP AND \\ RESULTS}

In this paper different graphs are generated using standard graph generator. The graphs are simple directed graphs with edge weights ranging from 1 to 12 . Reference solution to all the graphs is also calculated and compared with proposed single colony and multi colony solution.

Table 1 : values of parameters taken in proposed approaches

\begin{tabular}{|c|c|}
\hline PARAMETERS & VALUES \\
\hline $\boldsymbol{\alpha}$ & 1 \\
\hline $\boldsymbol{\beta}$ & 2 \\
\hline $\boldsymbol{\rho}$ & 0.5 \\
\hline
\end{tabular}

Table 2 : Results of single colony Max-Min approach

\begin{tabular}{|c|c|c|}
\hline $\begin{array}{c}\text { No. of vertices in } \\
\text { graph }\end{array}$ & $\begin{array}{c}\text { Tour length from } \\
\text { reference } \\
\text { solution }\end{array}$ & $\begin{array}{c}\text { Tour length from } \\
\text { single colony } \\
\text { Max-Min AS. }\end{array}$ \\
\hline 64 & 391 & 393 \\
\hline 128 & 896 & 899 \\
\hline 256 & 2294 & 2301 \\
\hline 512 & 3124 & 3131 \\
\hline 1024 & 6231 & 6238 \\
\hline 2048 & 14512 & 14528 \\
\hline
\end{tabular}

Table 3 : Results of Multi colony Max-Min approach

\begin{tabular}{|c|c|c|}
\hline $\begin{array}{c}\text { No. of vertices in } \\
\text { graph }\end{array}$ & $\begin{array}{c}\text { Tour length from } \\
\text { reference } \\
\text { solution }\end{array}$ & $\begin{array}{c}\text { Tour length from } \\
\text { single colony } \\
\text { Max-Min AS. }\end{array}$ \\
\hline 64 & 391 & 391 \\
\hline 128 & 896 & 897 \\
\hline 256 & 2294 & 2295 \\
\hline 512 & 3124 & 3124 \\
\hline 1024 & 6231 & 6233 \\
\hline 2048 & 14512 & 14514 \\
\hline
\end{tabular}

\section{COLCUSION}

In this paper single colony and multi colony approaches to Max-Min ant system is proposed. These approaches are applied for different graphs having vertex range from 64 to 2048. And the results are compared from reference solution. It is clear from analysis that the results obtained from multi colony ant system are better as compared to single colony.

\section{REFERENCES}

[1] Gautam Garai, Shayantan Debbarman, Tamalika Biswas "An Efficient Ant Colony Optimization Algorithm for Function Optimization" in IEEE 2013.

[2] Tianjun Liao, Krzysztof Socha, Marco A. Montes de Oca, Thomas Stutzle "Ant Colony Optimization for Mixed-Variable Optimization Problems" in IEEE TRANSACTIONS ON EVOLUTIONARY COMPUTATION, VOL. 18, NO. 4, AUGUST 2014.

[3] Ramlakhan Singh Jadon, Unmukh Datta "Modified Ant Colony Optimization Algorithm with Uniform Mutation using Self-Adaptive Approach for Travelling Salesman Problem" in IEEE 2013.

[4] Jerry Kponyo , Yujun Kuang , Enzhan Zhang "Dynamic Travel Path Optimization System Using Ant Colony Optimization" in IEEE 2014.

[5] Rafid Sagban, Ku Ruhana Ku Mahamud, Muhamad Shahbani Abu Bakar "Reactive Memory Model for Ant Colony Optimization and Its Application to TSP" in 2014 IEEE International Conference on Control System, Computing and Engineering, 28 - 30 November 2014, Penang, Malaysia.

[6] M. Veluscek, T. Kalganova, P. Broomhead "Improving Ant Colony Optimization Performance through Prediction of Best Termination Condition" in IEEE 2015.

[7] Zbigniew SWitnicki "Application of Ant Colony Optimization Algorithms for Transportation Problems Using the Example of the Travelling Salesman Problem" in 2015 4th IEEE International Conference on Advanced logistics and Transport (ICALT).

[8] Ms. K. Ilavarasi, Dr. K. Suresh Joseph "Variants of Travelling Salesman Problem: A Survey" in IEEE 2014.

[9] Morad BOUZIDI, Mohammed Essaid RIFF "Discrete Novel Hybrid Particle Swarm Optimization To Solve Travelling Salesman Problem" in IEEE 2014.

[10] Medhat A. Tawfeek, Ashraf El-Sisi, Arabi E. keshk, Fawzy A. Torkey "Cloud Task Scheduling Based on Ant Colony Optimization" in IEEE 2013. 